\title{
Impact of COVID-19 on Acute Invasive Fungal Rhinosinusitis: A Comparative Study
}

\author{
Original \\ Kamal Ebeid ${ }^{1}$,Mohamed Gamea ${ }^{2}$, Alzahraa A. Allam ${ }^{3}$ and Emad Shehata ${ }^{1}$ \\ Article \\ Department of Otorhinolaryngology, ${ }^{1}$ Faculty of Medicine, Tanta University, ${ }^{2}$ Misr University \\ for Science \& Technology, Faculty of Medicine, Egypt. \\ ${ }^{3}$ Department of Internal medicine, Faculty of Medicine Tanta University Hospital, Tanta, \\ Gharbia.
}

\begin{abstract}
Background: Acute invasive fungal rhinosinusitis (AIFR) is a potentially fatal infection that usually complicates immunosuppressive status like uncontrolled or newly discovered diabetes mellitus, during the coronavirus disease (COVID19) pandemic, worsening of underlying diabetes and newly discovered cases have been observed.

Aim: To highlight the impact of COVID-19 on emerging cases of AIFRS comparing this with AIFR before COVID-19 pandemic.

Methods: This is a retrospective comparative study between AIFR patients presenting to Tanta University Hospital, Egypt between July and December 2020 with a recent proven COVID-19 infection and AIFR patients presenting to the same institution prior to the covid-19 pandemic between January 2017 and December 2019.

Results: There was a dramatic increase in the rate of incidence of AIFR in patients with recent covid-19 infection in comparison with pre COVID-19 pandemic numbers. On the other hand, there was no statistical difference in the severity, extent of lesion or survival rate between the two groups.

Conclusion: A bidirectional relationship between Covid-19 and diabetes mellitus was observed together with immune dysregulation playing a possible role in subsequent increase in the rate of incidence of AIFR making it more emerging and more challenging.
\end{abstract}

Key Words: Acute invasive fungal rhinosinusitis, COVID-19, diabetic complications, rhinocerebral mucor mycosis, uncontrolled diabetes.

Received: 16 May 2021, Accepted: 05 August 2021

Corresponding Author: Kamal Ebeid, Department of Otorhinolaryngology, Faculty of Medicine, Tanta University, Egypt, Tel.: +20 1063898093, E-mail: Kamal.ebeid@med.tanta.edu.eg

ISSN: 2090-0740, 2021

\section{INTRODUCTION}

Invasive Fungal Rhinosinusitis is a profoundly serious medical condition which includes three subcategories, the acute invasive fungal rhinosinusitis, the chronic invasive fungal rhinosinusitis, and the rare chronic granulomatous invasive fungal rhinosinusitis ${ }^{[1]}$.

Theacuteinvasivefungalrhinosinusitis(AIFR)isahighly fatal disease that affects severely immunocompromised patients due to mycotic invasion of the mucosa of the nasal cavity and paranasal sinuses. Poorly controlled type 1 diabetes mellitus (DM), new chemotherapeutic agents and long-term use of immunosuppressive agents following bone marrow or solid organ transplantation, hematological malignancies, long standing multi organ failure have resulted in an increase in the population at risk of developing AIFR.
Multiple fungal species have been identified in patients with AIFR: Mucoraceae and Aspergillus are the most common pathogens ${ }^{[2]}$.

$\mathrm{DM}$ is well established as one of the major risk factors to develop severe degree of COVID-19 infection and to increase the mortality rate as well as development of new onset $\mathrm{DM}^{[3]}$.

The relationship between Covid-19 and diabetes could be described as a bidirectional multi risk relation. Patients with DM seem to be more prone to severe affection and a higher mortality rate. Patients with DM can be attributed to a facilitated viral uptake assisted by the host's receptor angiotensin-converting enzyme 2 (ACE2). It can also be associated with a higher basal level of pro-inflammatory cytokines present in patients with diabetes, which enables a hyperinflammatory "cytokine storm" in response to the virus. Also, an observed relation between the increased 
pro-inflammatory cytokines and insulin. Moreover, there is an observed poor control of DM in patients with covid 19 either due to the disease itself or the commonly used high doses of steroids which are routinely used in severe conditions to stop the disease process, which make the control of DM during covid 19 infection a real challenge $\mathrm{e}^{[4]}$.

This encouraged us to assess the impact of Covid-19 on the incidence of acute invasive fungal rhinosinusitis.

\section{PATIENTS AND METHODS}

\section{Ethical considerations}

This is study which was approved by the Ethical Committees of Faculty of Medicine, Tanta University, Egypt with approval code (34341/12/20). Confidentiality of the patients' data was warranted by keeping data anonymous (after assigning a code number to each patient that is known only by the investigators) and de-identification of images. Written consents were signed by patients or relatives.

\section{Study design}

This is a retrospective comparative study between AIFR patients presenting to Tanta University Hospital, Egypt between July and December 2020 with a recent proven COVID-19 infection and AIFR patients presenting to the same institution prior to the covid-19 pandemic between January 2017 and December 2019.

Diagnosis of AIFRS was based on history of recent manifestations of sinonasal infection, physical examination including meticulous endoscopic nasal examination, radiological modalities including coronal, axial, and sagittal CT scans, MRI, or both for sinonasal region in addition to histopathological assessment of nasal biopsies and fungal culture for identification of fungal species. Laboratory investigations to evaluate the general condition and diabetic control including fasting, 2hours post prandial blood sugar and glycosylated hemoglobin percentage.

The main stay of therapy is the proper diabetic control, all patients were admitted in ICU with close monitoring and proper control of diabetes. All patients were subjected to endoscopic Sino nasal tissue biopsies as office procedure for fungal and routine histopathological investigations, all patients were managed by amphotericin B infusion (Intravenous admixtures of amphotericin B 0.25 and 1.4 $\mathrm{mg} / \mathrm{mL}$ in $5 \%$ dextrose injection, beginning intravenous therapy with a 1-mg test dose. The usual daily maintenance dose of amphotericin $\mathrm{B}$ is $0.5-1 \mathrm{mg} / \mathrm{kg}$ i.e. The infusion was given over 4 hours with close monitoring of serum electrolytes and kidney functions).

Surgical debridement was done once the patients were candidate, debridement of all necrotic gangrenous elements was done either endoscopic, external, or combined endoscopic assisted, the limit of debridement was achieving apparent healthy surgical site.
Close daily examination of surgical site and debridement of any new necrotic elements till complete epithelialization of surgical site and histopathological confirmation of fungal free tissue biopsies at this time amphotericin B can be stopped.

\section{Statistical analysis of the data}

Data were fed to the computer and analyzed using IBM SPSS software package version 20.0. (Armonk, NY: IBM Corp). The Kolmogorov- Smirnov was used to verify the normality of distribution of variables, Comparisons between groups for categorical variables were assessed using Chi-square test (Fisher or Monte Carlo). Student t-test was used to compare two groups for normally distributed quantitative variables while Mann Whitney test was used to compare between two groups for not normally distributed quantitative variables. Significance of the obtained results was judged at the $5 \%$ level.

\section{RESULTS}

During 6 months period between July and December 2020, Tanta university hospitals admitted 28 AIFR patients. All of them gave history of a recent covid-19 infection proven by PCR and CT chest imaging that was diagnosed 2-8 weeks prior to the date of their presentation with AIFR. 22 patients (78.6\%) were known to be diabetic before their covid-19 infection, while 6 patients $(21.4 \%)$ were diagnosed with diabetes after their infection. All patients gave history of steroid administration in the form prednisolone 20-60 mg/day for 10-30 days as part of covid-19 treatment.

Both male and female patients represented $50 \%$ of this group of patients. The mean age was $41.2 \pm 15.8$ years. 7 patients $(25 \%)$ gave no history of comorbidities while 21 patients $(75 \%)$ had other comorbidities. 24 patients $(85.7 \%)$ had no history of thromboembolic manifestations.

On admission of those patients, The median fasting blood sugar was $215 \mathrm{mg} / \mathrm{dL}(150-400)$, The median 2 hour post prandial blood sugar was $405 \mathrm{mg} / \mathrm{dL}(300-614)$. The mean Glycosylated HB\% was $10.5 \pm 2.9$ patients (32.1\%) have no diabetic ketoacidosis. while 19 (67.9\%) patients had adiabatic ketoacidosis.

The median oxygen saturation was $89.5 \%(78-100)$. Severe lung affection of more than $40 \%$ was present in 7 patients $(25 \%)$. The median $\mathrm{C}$ reactive protein level was $60 \mathrm{mg} / \mathrm{L}(28-90)$. The mean serum ferritin level was $224.9 \pm 104.3$ microgram/L. The median LDH serum level was $461.5 \mathrm{U} / \mathrm{L}(234-890)$.

Histopathological assessment of nasal biopsies and fungal culture for those patients showed that Mucor species was identified in 19 patients (68\%) while Aspergillus species was found in 9 patients (32\%). (Figure 1) 


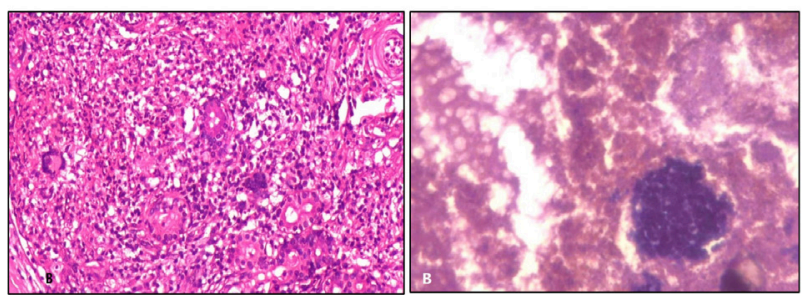

Fig. 1: Histopathological assessment of nasal biopsies showing mucosal and bony invasion by fungal colonies surrounded by dense inflammatory reactions

Encountered complications found through both examination and imaging included facial skin infarction in 15 patients $(53.6 \%$ ) (Figure 2), orbital affection with visual loss in 21 patients $(75 \%)$, intracranial affection with disturbed conscious level in 3 patients (10.7\%) (Figure 3), and cavernous sinus thrombosis was present in 11 patients $(39.3 \%)$.

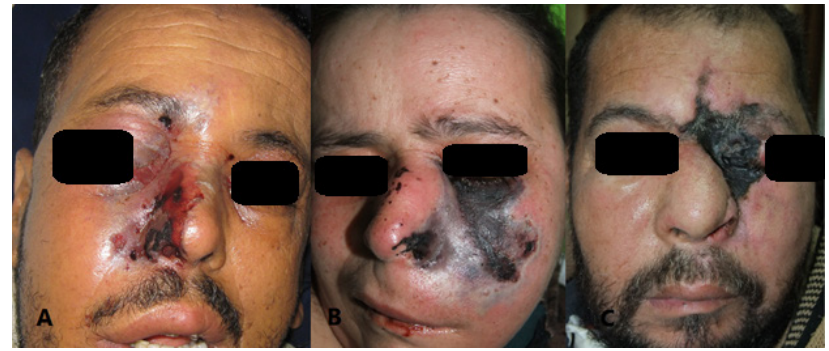

Fig. 2: Example of skin necrosis encountered with rhinocerebral mucormycosis
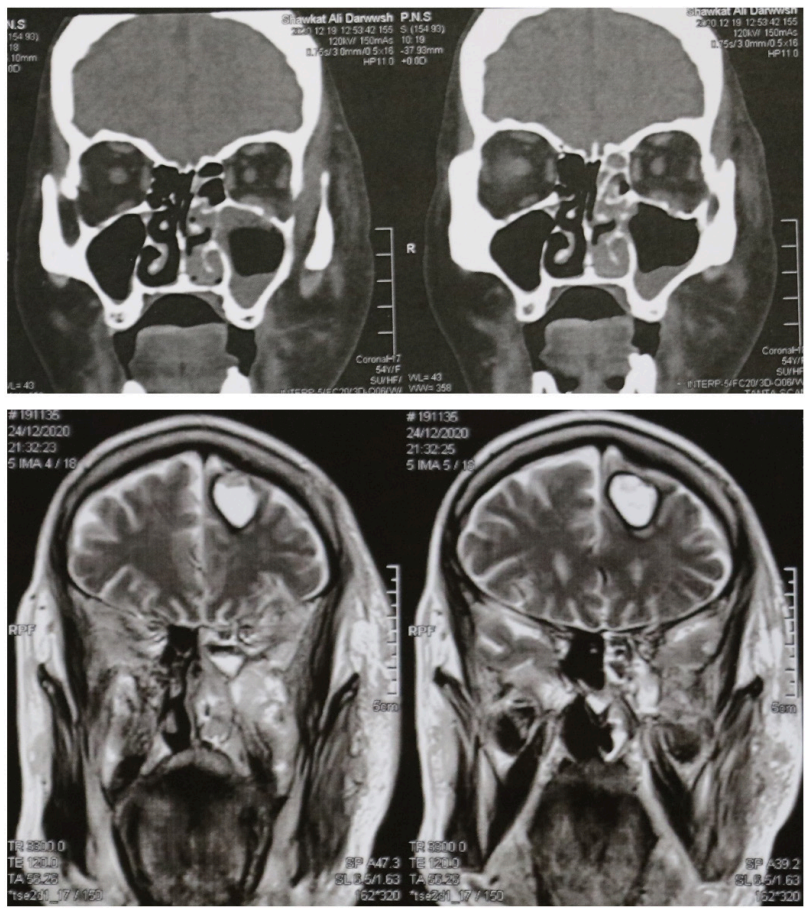

Fig. 3: A 54-year-old female patient with uncontrolled DM 1 month post COVID -19 infection presented with left facial pain and facial swelling A) CT scan of the nose and paranasal sinuses showing mucosal thickening of the left nasal cavity. B) MRI of this patient showing intracranial fungal abscess and marked swelling of the facial soft tissues
Surgical management varied according to the extent of infection. Hemi maxillectomy was done in 5 patients (17.9\%). Orbit exenteration was done in 7 patients $(25 \%)$. Endoscopic debridement was done in 7 patients $(25 \%)$ while external approach was adopted in 15 patients $(53.6 \%)$ and combined external and endoscopic approaches were done in 5 patients $(17.9 \%$ ). (Figures 4,5 ).
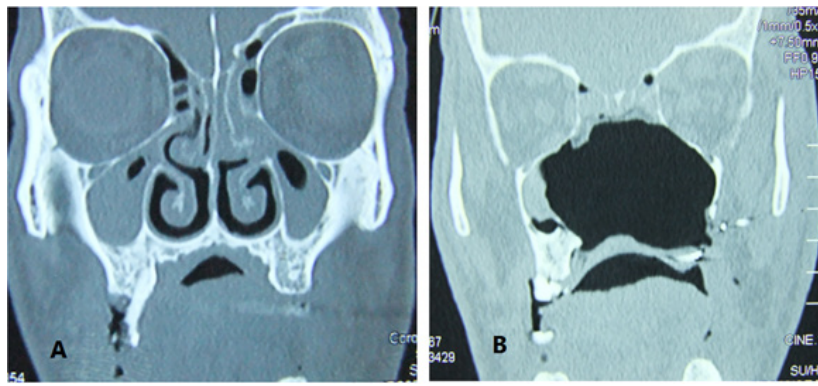

Fig. 4: A, pre-operative CT scan coronal cuts showing bilateral sinonasa tissues affection with AIFRS from second group of patients withou COVID-19 affection, B post-operative CT scan coronal cuts showing extensive debridement after 2 months.
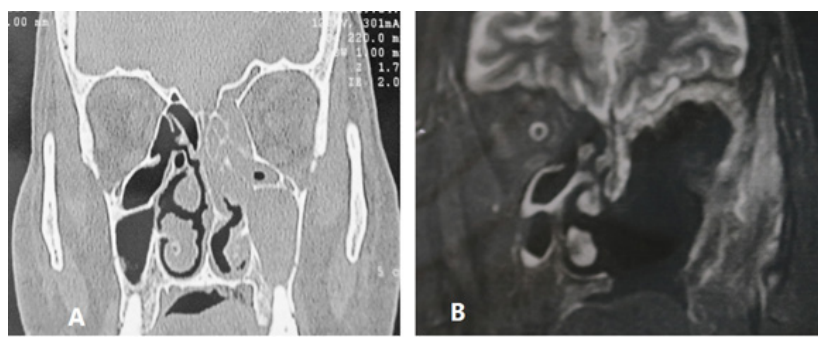

Fig. 5: A, pre-operative CT scan coronal cuts showing left side sinonasa tissues affection with AIFRS from first group of patients with COVID-19 affection, B post-operative MRI coronal cuts showing extensive debridement with maxillectomy and orbital exenteration cavity after 4 weeks

Proper diabetic control was achieved in 22 patients (78.6\%) using insulin infusion. Systemic intravenous Amphotericin B was administered in 25 patients (89.3\%). Thirteen patients died (46.4\%) in this group of patients.

On the other hand, during a 3 years period between January 2017 and December 2019, only 20 AIFR patients were admitted in Tanta university hospital, 7 male patients $(35 \%)$ and 13 females $(65 \%)$ with a mean age of $39.7 \pm$ 13.4 years. 4 patients $(20 \%)$ had no comorbidities while16 $(80 \%)$ had other comorbidities. 18 patients $(90 \%)$ gave no history of thromboembolic manifestations.

On admission, The median fasting blood sugar was 285 $\mathrm{mg} / \mathrm{dL}(170-400)$. The median 2 hour post prandial blood sugar was $411 \mathrm{mg} / \mathrm{dL}(300-614)$. The mean Glycosylated $\mathrm{HB} \%$ was $10.3 \pm 2 \mathrm{gm} / \mathrm{L} .6(30 \%)$ have no diabetic ketoacidosis. The median oxygen saturation was $99 \%$ $(89-100)$.

Histopathological assessment of nasal biopsies and fungal culture for the pre-covid group identified Mucor species in 13 patients $(65 \%)$ while Aspergillus species was found in 7 patients $(35 \%)$. 
Encountered complications that was found through examination and imaging included facial skin infarction in 11 patients $(55 \%)$, orbital affection with visual loss in 10 patients $(50 \%)$, intracranial extension with disturbed conscious level in 4 patients $(20 \%)$ and cavernous sinus thrombosis in 7 patients $(35 \%)$.

Surgical management included hemi maxillectomy in 4 patients (20\%). Orbital exenteration in 6 patients $(30 \%)$. Proper diabetic control was achieved in 16 patients $(80 \%)$. 8 patients died $((40 \%))$ in this group of patients.

\section{DISCUSSION}

This study represents the experience of a tertiary referral center (Tanta University Hospital, Egypt) with AIFRS during and prior to COVID19 pandemic.

According to our study, A recent dramatic increase in the rate of incidence of AIFR was noticed in comparison with pre COVID-19 pandemic numbers (Table 1,2). This may be explained by the impact of both COVID-19 infection and its associated steroid therapy on diabetic control. we even found out that 6 patients from the first group (21.4\%) were diagnosed with diabetes after covid-19 infection with no history of diabetes at all prior to the onset of covid-19 infection. These data strongly support the impact of COVID-19 on diabetic control and its bidirectional relationship as proposed by previous reports ${ }^{[5-7]}$ Also COVID-19 may be associated with increased risk of invasive fungal infection as reported by data from the UK National Mycology Reference Laboratory ${ }^{[8]}$. Also A dysregulated immune response to COVID-19 not only compromises the ability of the host to resolve the viral infection, but may also predispose the individual to secondary bacterial and fungal infections ${ }^{[9,10]}$.

There was no significant impact of age, sex, other comorbidities, and diabetic control between first and second group of patients, also we noticed that no significant difference between the two groups regarding clinical and radiological presentations this suggests that COVID-19 does not affect AIFR in a direct mechanism but through its impact on diabetic status with consequent immunosuppression, but this issue needs further large case volume studies.
Although there was a significant difference in oxygen desaturation between two groups at time of presentations, most of lung conditions were not affected in severe degree by COVID-19 to stage of severe hypoxemia to impact mortality rate in first group. There was no significant impact of COVID-19 on local extent of AIFRS disease and extent of surgical resection (Table 3 ).

Early complete surgical debridement with frozen section control and antifungal therapy are the cornerstones of AIFR management reversing the underlying immunosuppression altogether ${ }^{[11,12]}$.

According to our study results the survival rate was coinciding with that of the literature. With accordance to a systematic review on Fifty-two studies comprising a total of 807 patients, the overall survival rate was $49.7 \%^{[13]}$. but there was no significant difference between two groups of patients (Figure 6).

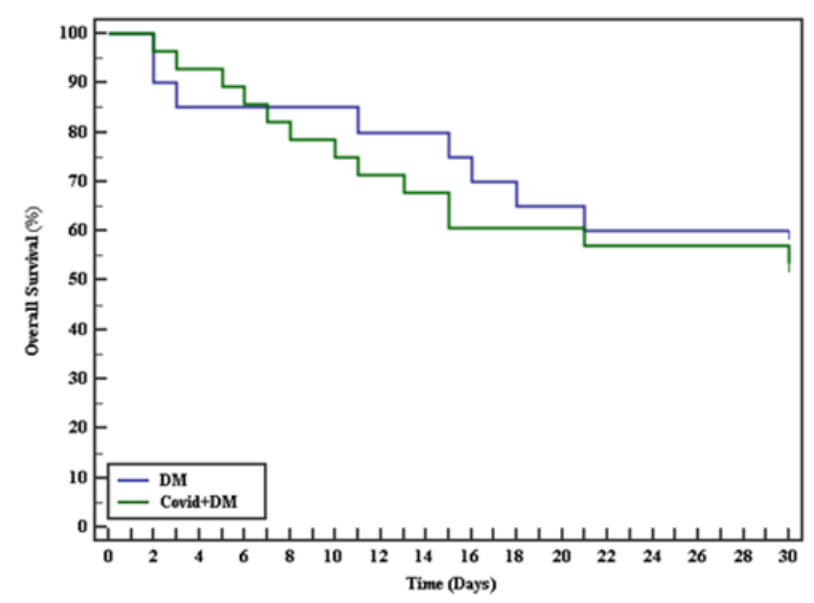

Fig. 6: Kaplan-Meier survival curve for overall Survival with groups

The main limitation of this study is the limited number of patients, also lack of postmortem examinations to defiantly detect mortalities etiologies.

In conclusion, ICU physicians, otolaryngologists should be aware that acute invasive fungal rhinosinusitis may be a fatal complication of critically ill COVID-19 patients and its early diagnosis and initiation of appropriate antifungal therapy for it is essential to reduce high mortality rate. 
Ebeid et. al.,

Table 1: Comparison between the two studied groups according to different parameters

\begin{tabular}{|c|c|c|c|c|}
\hline & $\begin{array}{c}\text { GROUP } 1(\text { COVID + DM) } \\
(\mathrm{n}=28)\end{array}$ & $\begin{array}{l}\text { GROUP } 2(\mathrm{DM}) \\
\quad(\mathrm{n}=20)\end{array}$ & Test of Sig. & $p$ \\
\hline \multicolumn{5}{|l|}{ Sex } \\
\hline Male & $14(50 \%)$ & $7(35 \%)$ & \multirow{2}{*}{$\chi^{2}=1.067$} & \multirow{2}{*}{0.302} \\
\hline Female & $14(50 \%)$ & $13(65 \%)$ & & \\
\hline \multicolumn{5}{|l|}{ Age (years) } \\
\hline Mean \pm SD & $41.2 \pm 15.8$ & $39.7 \pm 13.4$ & \multirow{2}{*}{$\mathrm{t}=0.340$} & \multirow{2}{*}{0.735} \\
\hline Median (Min. - Max.) & $43(12-67)$ & $41.5(16-65)$ & & \\
\hline No & $7(25 \%)$ & $4(20 \%)$ & \multirow[b]{2}{*}{$\chi^{2}=0.165$} & \multirow[b]{2}{*}{${ }^{\mathrm{FE}} \mathrm{p}=0.741$} \\
\hline Yes & $21(75 \%)$ & $16(80 \%)$ & & \\
\hline Hypertensive ischemic & $14(66.7 \%)$ & $11(68.8 \%)$ & $\chi^{2}=0.018$ & 0.893 \\
\hline Renal impairment & $6(28.6 \%)$ & $2(12.5 \%)$ & $\chi^{2}=1.384$ & ${ }^{\mathrm{FE}} \mathrm{p}=0.423$ \\
\hline COPD & $5(23.8 \%)$ & $4(25 \%)$ & $\chi^{2}=0.007$ & ${ }^{\mathrm{FE}} \mathrm{p}=1.000$ \\
\hline Hepatic & $1(4.8 \%)$ & $1(6.3 \%)$ & $\chi^{2}=0.039$ & ${ }^{\mathrm{FE}} \mathrm{p}=1.000$ \\
\hline \multicolumn{5}{|l|}{ History of DVT and thrombotic accidents } \\
\hline No history of thromboembolic manifestations & $24(85.7 \%)$ & $18(90 \%)$ & \multirow{2}{*}{$\chi^{2}=0.196$} & \multirow{2}{*}{${ }^{\mathrm{FE}} \mathrm{p}=1.000$} \\
\hline Positive history of thromboembolic manifestations & $4(14.3 \%)$ & $2(10 \%)$ & & \\
\hline \multicolumn{5}{|l|}{ Diabteic condition } \\
\hline Newly discovered DM with COVID & $6(21.4 \%)$ & $0(0 \%)$ & \multirow{2}{*}{$\chi^{2}=4.898^{*}$} & \multirow{2}{*}{${ }^{\mathrm{FE}} \mathrm{p}=0.034^{*}$} \\
\hline Known to be diabetic uncontrolled with COVID & $22(78.6 \%)$ & $20(100 \%)$ & & \\
\hline \multicolumn{5}{|l|}{ Fasting Bs } \\
\hline Mean \pm SD & $249 \pm 69.7$ & $261.4 \pm 68.7$ & \multirow{2}{*}{$\mathrm{U}=251.0$} & \multirow{2}{*}{0.543} \\
\hline Median (Min. - Max.) & $215(150-400)$ & $285(170-400)$ & & \\
\hline \multicolumn{5}{|l|}{$2 \mathrm{hPP}$ blood sugar } \\
\hline Mean \pm SD & $410.6 \pm 81.4$ & $417.3 \pm 88.3$ & \multirow{2}{*}{$\mathrm{t}=0.273$} & \multirow{2}{*}{0.786} \\
\hline Median (Min. - Max.) & $405(300-614)$ & $411(300-614)$ & & \\
\hline \multicolumn{5}{|l|}{ Diabetic ketoacidosis } \\
\hline No & $9(32.1 \%)$ & $6(30 \%)$ & \multirow{2}{*}{$\chi^{2}=0.025$} & \multirow{2}{*}{0.875} \\
\hline DKA present & $19(67.9 \%)$ & $14(70 \%)$ & & \\
\hline \multicolumn{5}{|l|}{ COVID 19 PCR } \\
\hline Recent positive PCR & $14(50 \%)$ & - & \multirow{2}{*}{-} & \multirow{2}{*}{-} \\
\hline Previous positive PCR within 2 months & $14(50 \%)$ & - & & \\
\hline \multicolumn{5}{|l|}{ Glycoselated HB\% (gm) } \\
\hline Mean \pm SD & $10.5 \pm 2$ & $10.3 \pm 2$ & \multirow{2}{*}{$\mathrm{t}=0.432$} & 0668 \\
\hline Median (Min. - Max.) & $10.5(7.5-14.4)$ & $10(7.5-13.9)$ & & 0.000 \\
\hline Preoperative radiology & & & & \\
\hline CT scan & $21(75 \%)$ & $14(70 \%)$ & & \\
\hline $\mathrm{CT}$ and MRI & $6(21.4 \%)$ & $5(25 \%)$ & $\chi^{2}=0.488$ & ${ }^{\mathrm{MC}} \mathrm{p}=1.000$ \\
\hline MRI only & $1(3.6 \%)$ & $1(5 \%)$ & & \\
\hline
\end{tabular}

$\chi^{2}$ : Chi square test MC: Monte Carlo FE: Fisher Exact t: Student t-test U: Mann Whitney test p: $p$ value for comparing between the studied groups.

*: Statistically significant at $p \leq 0.05$ 
Table 2: Comparison between the two studied groups according to different parameters

\begin{tabular}{|c|c|c|c|c|}
\hline & $\begin{array}{c}\text { GROUP } 1(\text { COVID + DM) } \\
(\mathrm{n}=28)\end{array}$ & $\begin{array}{l}\text { GROUP } 2(\mathrm{DM}) \\
(\mathrm{n}=20)\end{array}$ & Test of Sig. & $p$ \\
\hline Sinonasal tissues infarctions & $28(100 \%)$ & $20(100 \%)$ & - & - \\
\hline \multicolumn{5}{|l|}{ Skin infarction } \\
\hline No skin infarction & $13(46.4 \%)$ & $9(45 \%)$ & \multirow{2}{*}{$\chi^{2}=0.010$} & \multirow{2}{*}{0.922} \\
\hline Skin infarction present & $15(53.6 \%)$ & $11(55 \%)$ & & \\
\hline \multicolumn{5}{|l|}{ Orbital affection with visual loss } \\
\hline No affection & $7(25 \%)$ & $10(50 \%)$ & \multirow{2}{*}{$\chi^{2}=3.188$} & \multirow{2}{*}{0.074} \\
\hline Orbital affection & $21(75 \%)$ & $10(50 \%)$ & & \\
\hline \multicolumn{5}{|l|}{ Intracranial affection with disturbed conscious level } \\
\hline No affection & $25(89.3 \%)$ & $16(80 \%)$ & \multirow{2}{*}{$\chi^{2}=0.808$} & \multirow{2}{*}{${ }^{\mathrm{FE}} \mathrm{p}=0.429$} \\
\hline Intracranial affection & $3(10.7 \%)$ & $4(20 \%)$ & & \\
\hline \multicolumn{5}{|l|}{ Cavernous sinus thrombosis } \\
\hline No & $17(60.7 \%)$ & $13(65 \%)$ & \multirow{2}{*}{$\chi^{2}=0.091$} & \multirow{2}{*}{0.762} \\
\hline Present & $11(39.3 \%)$ & $7(35 \%)$ & & \\
\hline \multicolumn{5}{|l|}{$\mathrm{O}_{2}$ saturation at diagnosis $(\%)$} \\
\hline Median (Min. - Max.) & $10.5(7.5-14.4)$ & $10(7.5-13.9)$ & \multirow{2}{*}{$\mathrm{U}=68.500^{*}$} & \multirow{2}{*}{$<0.001^{*}$} \\
\hline Median (Min. - Max.) & $89.5(78-100)$ & $99(89-100)$ & & \\
\hline \multicolumn{5}{|l|}{ Initial c reactive protein $(\mathrm{mg} / \mathrm{L})$} \\
\hline Mean \pm SD & $60.6 \pm 17.5$ & $61.3 \pm 21.1$ & \multirow{2}{*}{$\mathrm{t}=0.130$} & \multirow{2}{*}{0.897} \\
\hline Median (Min. - Max.) & $60(28-90)$ & $60(28-105)$ & & \\
\hline \multicolumn{5}{|l|}{ Maxillectomy } \\
\hline Not done & $23(82.1 \%)$ & $16(80 \%)$ & \multirow{2}{*}{$\chi^{2}=0.035$} & \multirow{2}{*}{${ }^{\mathrm{FE}} \mathrm{p}=1.000$} \\
\hline Done & $5(17.9 \%)$ & $4(20 \%)$ & & \\
\hline \multicolumn{5}{|l|}{ Orbit exenteration } \\
\hline Not done & $21(75 \%)$ & $14(70 \%)$ & \multirow{2}{*}{$\chi^{2}=0.148$} & \multirow{2}{*}{0.701} \\
\hline Done & $7(25 \%)$ & $6(30 \%)$ & & \\
\hline \multicolumn{5}{|l|}{ Diabetic control } \\
\hline Controlled & $22(78.6 \%)$ & $16(80 \%)$ & \multirow{2}{*}{$\chi^{2}=0.014$} & \multirow{2}{*}{${ }^{\mathrm{FE}} \mathrm{p}=1.000$} \\
\hline Poor control & $6(21.4 \%)$ & $4(20 \%)$ & & \\
\hline \multicolumn{5}{|l|}{ Systemic antifungal } \\
\hline Patient not received antifungal treatment due to death & $3(10.7 \%)$ & $3(15 \%)$ & \multirow{2}{*}{$\chi^{2}=0.196$} & $\mathrm{FE}_{\mathfrak{n}}=0.683$ \\
\hline Systemic amphotericin B & $25(89.3 \%)$ & $17(85 \%)$ & & $p-0.003$ \\
\hline Outcome & & & & \\
\hline Died & $13(46.4 \%)$ & $8(40 \%)$ & $\chi^{2}=0.196$ & 0.658 \\
\hline Survived & $15(53.6 \%)$ & $12(60 \%)$ & $\chi-0.190$ & 0.050 \\
\hline
\end{tabular}

$\chi^{2}$ : Chi square test MC: Monte Carlo FE: Fisher Exact t: Student t-test U: Mann Whitney test p: $p$ value for comparing between the studied groups. *: Statistically significant at $p \leq 0.05$ 
Table 3: Distribution of the studied cases according to different parameters COVID + DM $(n=28)$

\begin{tabular}{|c|c|}
\hline & No. (\%) \\
\hline \multicolumn{2}{|l|}{ Lung affection degree of infiltration } \\
\hline No lung affection & $4(14.3 \%)$ \\
\hline $\begin{array}{l}\text { Mild lung affection with COVID less than } \\
20 \%\end{array}$ & $13(46.4 \%)$ \\
\hline Moderate $20-40 \%$ & $4(14.3 \%)$ \\
\hline Severe more than $40 \%$ & $7(25 \%)$ \\
\hline \multicolumn{2}{|l|}{ Serum ferritin micrograms $(\mathrm{L})$} \\
\hline Mean \pm SD & $224.9 \pm 104.3$ \\
\hline Median (Min. - Max.) & $239.5(56-456)$ \\
\hline \multicolumn{2}{|l|}{ LDH serum level (U/L) } \\
\hline Mean \pm SD & $507.6 \pm 153$ \\
\hline Median (Min. - Max.) & $461.5(234-890)$ \\
\hline \multicolumn{2}{|c|}{$\begin{array}{l}\text { Initial dose of steroid used for COVID } 19 \text { before and at the } \\
\text { presentation }\end{array}$} \\
\hline Mean \pm SD & $32.9 \pm 9$ \\
\hline Median (Min. - Max.) & $40(20-40)$ \\
\hline \multicolumn{2}{|l|}{ Surgical approach } \\
\hline $\begin{array}{l}\text { Patient not received surgical treatment due } \\
\text { to death }\end{array}$ & $1(3.6 \%)$ \\
\hline Endoscopic debridement & $7(25 \%)$ \\
\hline Debridement through external approach & $15(53.6 \%)$ \\
\hline $\begin{array}{l}\text { Combined external and endoscopic } \\
\text { debridement }\end{array}$ & $5(17.9 \%)$ \\
\hline
\end{tabular}

\section{Data availability statement}

The data supporting the findings of this study are available from the corresponding author upon reasonable request.

\section{CONFLICT OF INTERESTS}

There are no Conflicts of Interest.

\section{REFERENCES}

1. Süslü AE · Öfretmenoflu O S Süslü N· Yücel OT Önerci TM: Acute invasive fungal rhinosinusitis: our experience with 19 patients. EurArch Otorhinolaryngol (2009) 266:77-82

2. deShazo RD, O’Brien M, Chapin K, Soto-Aguilar M, Gardner L,Swain R (1997) A new classiWcation and diagnostic criteria for invasive fungal sinusitis. Arch Otolaryngol Head Neck Surg 123:1181-1188

3. Moazzami B, Chaichian S, Kasaeian A, Djalalinia S, Akhlaghdoust M, Eslami M, Broumand B. Metabolic risk factors and risk of Covid-19: A systematic review and meta-analysis. PLoS One. 2020 Dec 15;15(12): e0243600. doi: 10.1371/journal.pone.0243600 PMID: 33320875.

4. 4-Azar WS, Njeim R, Fares AH, et al. COVID-19 and diabetes mellitus: how one pandemic worsens the other. Rev Endocr Metab Disord. 2020;21(4):451-463. doi:10.1007/s11154-020-09573-6

5. Li J, Wang X, Chen J, Zuo X, Zhang H, Deng A. COVID-19 infection may cause ketosis and ketoacidosis. Diabetes Obes Metab 2020 April 20 (Epub ahead of print).

6. Ren H, Yang Y, Wang F, et al. Association of the insulin resistance marker TyG index with the severity and mortality of COVID-19. Cardiovasc Diabetol 2020; 19: 58 .

7. Hamming I, Timens W, Bulthuis ML, Lely AT, Navis $\mathrm{G}$, van Goor H. Tissue distribution of ACE2 protein, the functional receptor for SARS coronavirus: a first step in understanding SARS pathogenesis. J Pathol 2004; 203: 631-7.

8. Borman AM, Palmer MD, Fraser M, Patterson Z, Mann C, Oliver D, Linton CJ, Gough M, Brown P, Dzietczyk A, Hedley M, McLachlan S, King J, Johnson EM. COVID-19-Associated Invasive Aspergillosis: Data from the UK National Mycology Reference Laboratory. J Clin Microbiol. 2020 Dec 17;59(1)

9. Costantini C, van de Veerdonk FL, Romani L. Covid-19-Associated Pulmonary Aspergillosis: The Other Side of the Coin. Vaccines (Basel) 2020 Dec 1;8(4):713.

10. Hoenigl, M. Invasive Fungal Disease complicating COVID-19: When it rains it pours. Clin. Infect. Dis. 2020 .

11. Zuniga, Maria G.; Turner, Justin H. Treatment outcomes in acute invasive fungal rhinosinusitis, Current Opinion in Otolaryngology \& Head and Neck Surgery: June 2014 - Volume 22 - Issue 3 - p 242-248.

12. Craig, John R. Updates in management of acute invasive fungal rhinosinusitis, Current Opinion in Otolaryngology \& Head and Neck Surgery: February 2019 - Volume 27 - Issue 1 - p 29-36.

13. Turner JH, Soudry E, Nayak JV, Hwang PH. Survival outcomes in acute invasive fungal sinusitis: a systematic review and quantitative synthesis of published evidence. The laryngoscope. 2013 May;123(5):1112-8. 